\title{
Tridimensional assessment of the dental follicle dimensions of impacted mandibular third molars using cone-beam CT
}

\author{
Marlene Barroso ${ }^{1}$, Luis-Ernesto Arriola-Guillén ${ }^{1,2}$, Yalil-Augusto Rodríguez-Cárdenas ${ }^{1,3}$, Gustavo-Armando \\ Ruíz-Mora ${ }^{1,4}$, Maria-Eugenia Guerrero ${ }^{1}$, Carlos Flores-Mir ${ }^{5}$
}

\footnotetext{
${ }^{1}$ Division Oral and Maxillofacial Radiology, School of Dentistry, Universidad Científica Sur, Lima, Perú

${ }^{2}$ Division of Orthodontics, School of Dentistry, Universidad Científica del Sur, Lima, Perú

${ }^{3}$ Division of Oral and Maxillofacial Radiology, Faculty of Dentistry, Universidad Nacional de Colombia, Bogotá, Colombia

${ }^{4}$ Division of Orthodontics, Faculty of Dentistry, Universidad Nacional de Colombia, Bogotá, Colombia

${ }^{5}$ Department of Dentistry, Faculty of Medicine and Dentistry, University of Alberta, Edmonton, Canada
}

Correspondence:

Av. Paseo de la República $N^{\circ} 5544$

Lima 18-Perú

mguerrero@cientifica.edu.pe

Received: $23 / 08 / 2017$ Accepted: $23 / 12 / 2017$

Barroso M, Arriola-Guillén LE, Rodríguez-Cárdenas YA, Ruíz-Mora GA, Guerrero ME, Flores-Mir C. Tridimensional assessment of the dental follicle dimensions of impacted mandibular third molars using conebeam CTh. J Clin Exp Dent. 2018;10(8):e726-31.

http://www.medicinaoral.com/odo/volumenes/v10i8/jcedv10i8p726.pdf

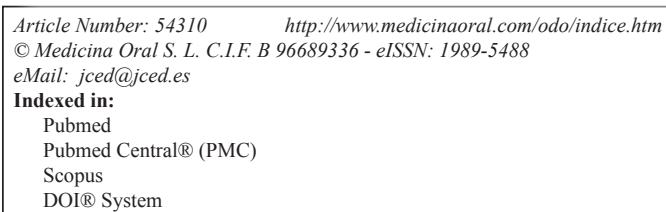

\begin{abstract}
Background: The present study was performed to compare follicle dimensions of impacted mandibular third molar (IMTM) with different impaction angulations using cone beam computed tomography (CBCT).

Material and Methods: Forty-nine individuals with IMTM (24 male, 25 female) were selected. Their age range was 25-55 years. The sample was divided into three IMTM groups either vertical $(n=16)$, mesioangular $(n=18)$ or horizontal $(n=15)$ position based on Winter's classification (the angle between the longitudinal axis of the second and third molars). Follicular spaces (FS) from available CBCT imaging were measured from the midpoint of the teeth's crown in several dimensions (mesial, distal, occlusal, apical, vestibular and lingual) in axial, sagittal and coronal planes. An ANOVA, T-student, Kruskal-Wallis and Mann-Whitney U tests were used.

Results: A comparison of the mesial FS for all groups revealed significant differences $(p<0.001)$. Significant difference was also found for vestibular FS between the vertical and mesioangular IMTM groups $(p=0.04)$. Buccolingual FS for all groups revealed no significant differences $(p=0.074)$, whereas significant difference was found for the vertical and horizontal IMTM groups $(p=0.02)$. No significant statistical differences were found for occlusal $(p=0.54)$, apical $(p=0.06)$, and lingual $(p=0.64) \mathrm{FS}$.

Conclusions: In this sample IMTM follicles have different dimensions according to their degree of angulation. Mesioagulated and horizontally positioned IMTMs seems to consistently have some increased FS dimensions (mesial and vestibular aspects).
\end{abstract}

Key words: Dental follicle, impacted tooth, third molar, cone-beam computed tomography. 


\section{Introduction}

An impacted tooth can be defined as a tooth that is unable to erupt into its position within its expected eruption time because of malposition or lack of space (1). Third molars are the most frequently impacted teeth $(2,3)$. They do normally erupt sometime between 18 and 25 years of age (4). The prevalence of third molar impaction ranges from $16.7 \%$ to $68.6 \%$ (5-7). Most studies have reported a higher frequency in females than males (8-11).

The dental follicle (DF) around impacted teeth has potential to develop pathological conditions $(12,13)$. Radiographically, the DF appears as a thin pericoronal radiolucency considered normal when it is less than $3 \mathrm{~mm}$ thick (12-18). According to the recommendations of National Institute of Health (NIH) both impacted and erupted mandibular third molars with evidence of follicular enlargement should be considered for extraction and the associated soft tissue submitted for microscopic examination (19). It has to be noted that there has not been an internationally accepted consensus on radiographic criteria to differentiate between normal and abnormal conditions of follicular tissue around impacted third molars (14).

A significant part of the problem is related to different magnification rates and spatial tissue overlap expected in either analog or digital two-dimensional radiography (15). It has been speculated that CBCT imaging could be more accurate than conventional two-dimensional radiographic methods in displaying third molar morphology and its related anatomical structures (17). However, CBCT imaging must be properly justified for each patient and should only be requested when there is a potential to provide new information that can impact clinical management decisions not offered by conventional radiography $(15,18)$.

The most frequently observed third molar impaction is a mesioangular position, with a relative frequency of 50 $\%$. There is some controversy regarding how the impaction position may be associated to an increased pathological risk. Some claimed that an horizontally position tooth tends to be the most affected by pathological changes (20), while others maintained that there is no evidence that intraosseous position of a impacted mandibular third molar (IMTM) could lead to an increased risk of developing a cyst or tumor (21-24). It has also been suggested that if a more accurate assessment of its angulation could be provided then this information could serve as an additional tool to justify treatment management decisions (21).

Neither the thickness of the follicle nor the associated three-dimensional positions of the mandibular third molars have been reported yet through CBCT imaging on cases with strong suggestion of impaction. Therefore, the purpose of this study was to compare follicle dimen- sions of impacted mandibular third molar with different impaction angulations (mesioangular, vertical or horizontal positions) through cone beam computed tomography (CBCT). We tried to refuse the null hypothesis that there are not differences in the follicle dimension between different angulations of impacted third molar.

\section{Material and Methods}

\section{-Sample characteristics}

This cross-sectional study was approved by the ethical committee of the School of Dentistry, Científica del Sur University-Lima, Perú, ( $\mathrm{N}^{\circ}$ 000227). This study included 49 CBCTs (24 male, 25 female) with impacted mandibular third molar (IMTM) previously taken for reasons not related to this study at CDI Diagnostic Imaging Center in Lima, Perú. Their age range was 25-55 years (mean age 28.6 years old). The sample was divided into three IMTM groups either vertical $(n=16)$, mesioangular $(n=18)$ or horizontal $(n=15)$ position based on Winter's classification (the angle between the longitudinal axis of the second and third molars). The sample size calculation was determined considering a mean difference of $1.5 \mathrm{~mm}$ in the mesial dimensions of the follicular spaces as a clinically relevant difference between impacted third molar with vertical and horizontal angulations. A standard deviation of $1 \mathrm{~mm}$ was considered (obtained from a preliminary pilot study) with a two-sided significance level of 0.05 and a power of $80 \%$. Although a minimum of 6 IMTM was required, a minimum of 15 IMTM were available.

The inclusion criteria consisted on the following:

1) CBCT scans taken with the same machine under the same parameters from January 2014 to December 2014; 2) Participants aged between 25 and 55 years;

3) IMTM in either vertical, mesioangular or horizontal position based on Winter's classification (21) (the angle between the longitudinal axis of the second and third molars is categorized as follows: Vertical impaction: $10^{\circ}$ to $-10^{\circ}$; mesioangular impaction: $11^{\circ}-79^{\circ}$; horizontal impaction: $80^{\circ}-100^{\circ}$ ) (Fig. 1);

4) IMTM class C based on Pell y Gregory's classification (the relation of the cementoenamel junction (CEJ) of the third molar with the bone level is categorized as follows: Level A: Not buried in bone; level B: Partially buried in bone if any part of CEJ was lower than bone level; level C: Completely buried in bone);

5) IMTM with complete root formation.

Exclusion criteria were as follows:

1) IMTM class A and class B (Pell y Gregory's classification);

2) Presence of any pathological and developmental conditions in the area surrounding the IMTM (i.e., tumors, cysts, fractures, or malformations);

3) Obvious alterations in size and in shape of IMTM, and 

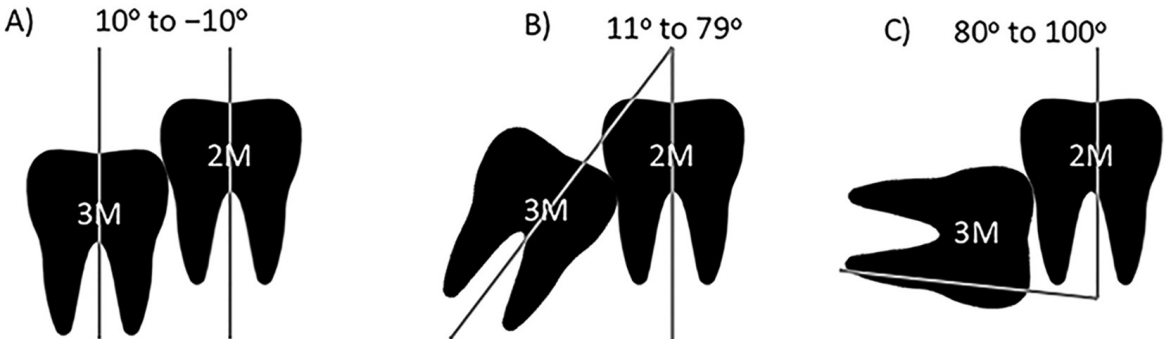

Fig. 1: IMTM in vertical, mesioangular or horizontal position. A) Vertical impaction: $10^{\circ}$ to $-10^{\circ}$; B) Mesioangular impaction: $11^{\circ}-79^{\circ}$; C) Horizontal impaction: $80^{\circ}-100^{\circ}$.

4) Presence of any artifacts or blurring affecting image quality around the are of interest.

-Cone beam computed tomography examination Imaging was performed with a Picasso Master 3D (Vatech, E-WOO Technology Co, Ltd, Republic of Korea) CBCT machine. Device settings were set at $8 \mathrm{~mA}$ and 90 $\mathrm{kV}$. Each field of view (FOV) mode was $20 \mathrm{~cm} \times 19 \mathrm{~cm}$, and with an isotropic voxel size of $0.4 \mathrm{~mm}$.

Participants were in centric occlusion (maximum intercuspidation) during $\mathrm{CBCT}$ imaging. The $\mathrm{CBCT}$ volumetric data sets (one for each mandibular third molar region) were reconstructed in three planes: axial, sagittal and coronal. The data sets were viewed using EZ Implant 3D software v.1.5 (Vatech, E-WOO Technology Co, Ltd, Republic of Korea). All images were assessed in a dark room on one monitor (S19C150 Samsung 18.5 inch flat panel liquid-crystal display monitor - SAMSUNG, South Korean) set at a resolution of $1366 \times 768$ pixels. The subject's head scan was positioned based on the Frankfurt plane (Po-Or). Follicular spaces (FS) were measured from the midpoint of the teeth's crown in several dimensions (mesial, distal, occlusal, apical, vestibular and lingual) in axial, sagittal and coronal planes (Fig. 2). All patients were informed in advance that the scans might be anonymously used for research reasons later and their consent was obtained. An intra-examiner calibration procedure consisted on the primary investigator measuring 5 pairs of CBCT images 2 times after one week. Intra-examiner reliability was assessed with the intraclass correlation coefficient (ICC).

-Statistical evaluation

Statistical analyses were performed by transferring all the data on Microsoft Excel 2011 software (Microsoft Corporation) and the Statistical Package for the Social Sciences software SPSS v.21 for MAC (IBM SPSS, Armonk, NY: IBM Corp.) was used. Normal distribution was confirmed by Shapiro-Wilk tests in some groups (coronal). A one-way analysis of variance (ANOVA) test and several t-tests were performed to determine whether there were differences in diameter of FS in the six measured dimensions and according to IMTM angulation. The equivalent non-parametric Kruskal-Wallis test and Mann-Whitney U-test was used when normality was not satisfied in some groups (vestibular, lingual, mesial, distal, apical). Statistical significance was set at $p<0.05$ for all tests.

\section{Results}

Intra-examiner reliability gave a result greater than 0.90 for all measurements (confidence intervals between $0.900-0.998$ ).

Descriptive statistics were used to summarize the sample characteristics according to IMTM angulation, sex and age. A comparison of the mesial FS for all groups re-

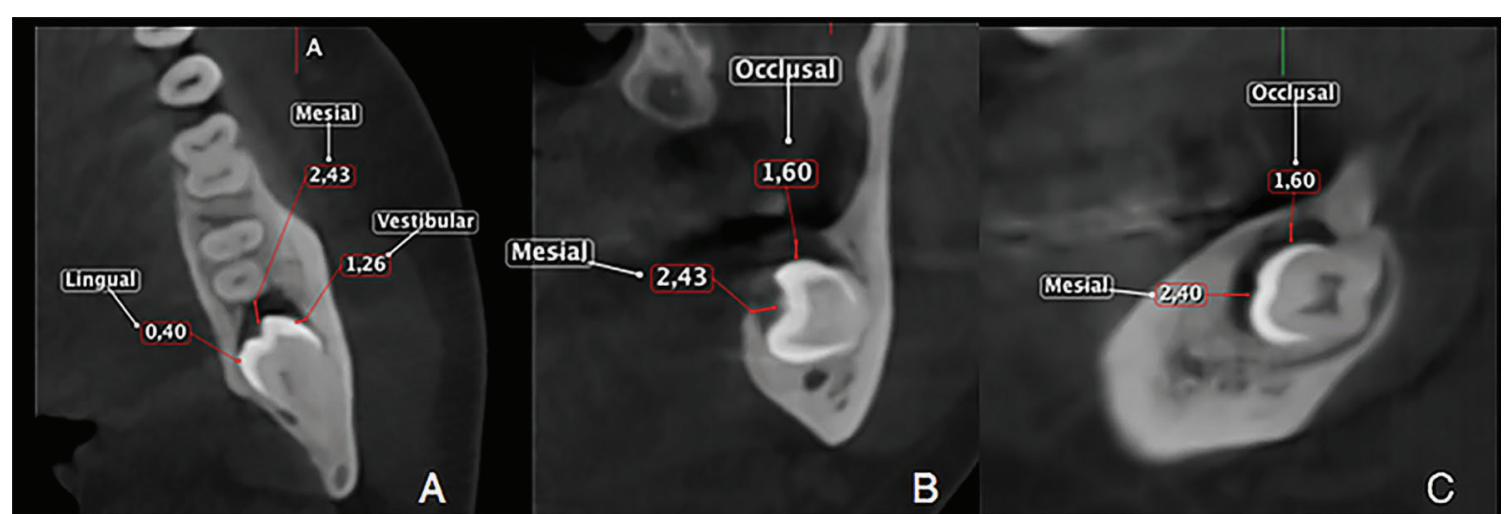

Fig. 2: FS measurements performed on different CBCT sections. A. Axial view showing vestibular, mesial and lingual FS measurements on a horizontal impacted third molar B. Vestibular, occlusal and lingual FS measurements performed on a coronal view C. Sagittal view of the FS occlusal measurement. 
vealed significant differences $(p<0.001)$ (Table 1). Moreover, significant difference was found for vestibular FS between the vertical and mesioangular IMTM groups $(p=0.04)$ (Table 2). However, buccolingual FS for all groups revealed no significant differences $(p=0.074$ ), whereas significant difference was found for the vertical and horizontal IMTM groups $(p=0.02)$ (Table 3 ). No significant statistical differences was found for occlusal ( $p=0.54)$, apical $(p=0.06)$, and lingual $(p=0.64) \mathrm{FS}$ (Table 4).

\section{Discussion}

The presence of apparent impacted mandibular third molars is a common finding in routine dental examinations $(2,3)$. There is a controversy on whether a small pericoronal radiolucent image may be an indicator of future pathological changes which may support the need for a prophylactic extraction $(3,24)$. There have been studies $(12,13)$ that have investigated impacted mandibular third molars follicles with two-dimensional radiographic imaging and their association with histologically deter-

Table 1: Comparison of the mesial dimensions of the follicular spaces depending on the molar position.

\begin{tabular}{|c|c|c|c|c|c|}
\hline Position & n & Mean & SD & Min & Max \\
\hline (1) Vertical & 16 & 0.85 & 0.40 & 0.30 & 1.90 \\
\hline (2) Mesioangular & 18 & 2.78 & 1.52 & 0.60 & 5.70 \\
\hline (3) Horizontally & 15 & 2.14 & 1.19 & 0.70 & 4.70 \\
\hline \multicolumn{6}{|c|}{$\begin{array}{l}p<0.001 \text { Kruskal-Wallis test } \\
1 \text { Y } 2 p<0.001 \text { Mann-Whitney U test } \\
1 \text { Y } 3 p<0.001 \text { Mann-Whitney U test } \\
2 \text { Y } 3 p=0.259 \text { Mann-Whitney U test }\end{array}$} \\
\hline
\end{tabular}

Table 2: Comparison of the vestibular dimensions of the follicular spaces depending on the molar position.

\begin{tabular}{|c|c|c|c|c|c|}
\hline Position & $\mathbf{n}$ & Mean & SD & Min & $\operatorname{Max}$ \\
\hline (1) Vertical & 16 & 0.61 & 0.17 & 0.20 & 0.90 \\
\hline (2) Mesioangular & 18 & 1.12 & 1.42 & 0.50 & 6.80 \\
\hline (3) Horizontally & 15 & 0.76 & 0.43 & 0.20 & 1.60 \\
\hline
\end{tabular}

Table 3: Comparison of the distal dimensions of the follicular spaces depending on the molar position.

\begin{tabular}{|l|c|c|c|c|c|}
\hline Position & $\mathbf{n}$ & Mean & SD & Min & Max \\
\hline (1) Vertical & 16 & 11.64 & 0.79 & 10.20 & 13.00 \\
\hline (2) Mesioangular & 18 & 12.26 & 2.03 & 9.80 & 19.20 \\
\hline (3) Horizontally & 15 & 12.56 & 1.09 & 10.70 & 15.30 \\
\hline
\end{tabular}

$p=0.074$ Kruskal-Wallis test

1 vs. $2 p=0.30$ Mann-Whitney U test

1 vs. $3 p=0.02$ Mann-Whitney U test

2 vs. $3 p=0.20$ Mann-Whitney $U$ test 
Table 4: Comparison of the lingual, occlusal and apical dimensions of the follicular spaces depending on the molar position.

\begin{tabular}{|l|c|c|c|c|c|c|c|}
\hline $\begin{array}{c}\text { Follicular } \\
\text { spaces }\end{array}$ & Position & $\mathbf{n}$ & Mean & SD & Min & Max & $p$ value \\
\hline Lingual & Vertical & 16 & 0.63 & 0.30 & 0.10 & 1.30 & \\
\hline & Mesioangular & 18 & 0.71 & 0.29 & 0.10 & 1.30 & $0.64^{*}$ \\
\hline & Horizontally & 15 & 0.78 & 0.68 & 0.30 & 3.20 & \\
\hline Occlusal & Vertical & 16 & 1.59 & 0.85 & 0.10 & 2.90 & \\
\hline & Mesioangular & 18 & 1.38 & 0.70 & 0.60 & 3.20 & $0.54^{* *}$ \\
\hline & & & & & & & \\
\hline & Horizontally & 15 & 1.30 & 0.73 & 0.10 & 2.70 & \\
\hline & & & & & & & 0 \\
\hline Apical & Mesioangular & 18 & 0.92 & 0.21 & 0.50 & 1.30 & $0.06^{* * *}$ \\
\hline
\end{tabular}

* Kruskal-Wallis test

**ANOVA test

*** Mann-Whitney U test

mined findings. Their findings suggested that follicles larger than $2.5 \mathrm{~mm}$ could have increased risk for developing associated pathology.

Cone beam computed tomography (CBCT) has been validated in several studies as an accurate and reliable measurement method for craniofacial structures overall. This is at least in part due to the fact that CBCT images consist of isotropic voxels (equal in length, height and length), which enable geometrically accurate measurements in any plane of space $(17,25)$. The impact of this imaging technique on dental treatment planning has also been discussed before (26). In addition, it has been suggested that it is possible to obtain coronal, sagittal, and trans-axial reconstructions in 3D and conventional two-dimensional images from an axial CBCT slice (27). These options make it possible to make a more accurate diagnosis for certain treatment management decisions. To our best knowledge there are no published studies that have quantified FS in mandibular impacted third molars with different angulations using $\mathrm{CBCT}$ imaging. The current results showed statistically significant differences in the vestibular FS dimensions between the third molars groups that were classified as in vertical or mesioangular impacted position. The results also showed statistically significant differences in the mesial FS between the group of third molars in vertical position and those of third molars in mesioangular and horizontal positions. This suggests that mandibular impacted third molars in mesioangular and horizontal positions are more predisposed to develop larger sized FS in certain directions and the likelihood to create a cyst will be increased. Similar results shown that the dimensions of the DF could depend on the mandibular morphology and associated areas of smaller bone resistance. Leitner et al. stated that the horizontal position of impacted mandibular third molars tended to be more affected by pathological changes (20). However, Haghanifar et al. could not identify a significant association between the DF diameter, the mesiodistal width of the dental crown and the histopathological evaluation (25). It was concluded that the diameter of the DF and the mesiodistal wi$\mathrm{dth}$ of the teeth cannot be used as a diagnostic index for differentiating between a normal and pathological DF. It has to be noted that several studies $(28,29)$, recommended prophylactic extraction by other reasons not linked to the DF size, such as the risk of caries and periodontal disease on the adjacent second molar. However, there are not studies to demonstrate the variation of the dental follicle in relation to the angulation of impacted mandibular third molar.

It is suggested to carry out future studies with the aim of establishing reference values of the DF spaces of impacted third molars evaluated through CBCT imaging and their association with an increased likelihood of the presence of (or progression to) a histologically diagnosed cystic or tumor lesion. In cases with a higher pathology development risk prophylactic actions to prevent or decrease the associated adverse effects may be justified.

A reference standard measurement for the FS of normally erupting third molars are lacking. Finally, we refuse the null hypothesis and conclude that the follicles of impacted third molars have different dimensions according 
to their degree of angulation. Mesioangulated and horizontally positioned IMTMs seems to consistently have some increased FS dimension.

\section{Conclusions}

In this sample IMTM follicles have different dimensions according to their degree of angulation. Mesioangulated and horizontally positioned IMTMs seems to consistently have some increased FS dimensions (mesial and vestibular aspects). No reference standard FS dimensions are available.

\section{References}

1. Rantanan A. The age of eruption of third molar teeth. Acta Odontol Scand. 1974:44:141-145.

2. Khawaja NA, Khalil H, Parveen K, Al-Mutiri A, Al-Mutiri S, AlSaawi A. A retrospective radiographic survey of pathology associated with impacted third molars among patients seen in Oral \& Maxillofacial Surgery Clinic of College of Dentistry, Riyadh. J Int Oral Health. 2015;7:13-17.

3. Stathopoulos P, Mezitis M, Kappatos C, Titsinides S, Stylogianni E. Cysts and tumors associated with impacted third molars: is prophylactic removal justified? J Oral Maxillofac Surg. 2011;69:405-408.

4. Kaushal N. Is radiographic appearance a reliable indicator for the absence or presence of pathology in impacted third molars? Indian $\mathrm{J}$ Dent Res. 2012;23:298-302.

5. Kaya GS, Aslan M, Ömezli MM, Dayi E. Some morphological features related to mandibular third molar impaction. J Clin Exp Dent. 2010;2:e12-e7.

6. Hashemipour MA, Tahmasbi-Arashlow M, Fahimi-Hanzaei F. Incidence of impacted mandibular and maxillary third molars: a radiographic study in a Southeast Iran population. Med Oral Patol Oral Cir Bucal. 2013;18:40-45.

7. Quek SL, Tay CK, Tay KH, Toh SL, Lim KC. Pattern of third molar impaction in a Singapore Chinese population: a retrospective radiographic survey. Int J Oral Maxillofac Surg. 2003;32:548-552.

8. Kruger E, Thomson WM, Konthasinghe P. Third molar outcomes from age 18 to 26: findings from a population-based New Zealand longitudinal study. Oral Surg Oral Med Oral Pathol Oral Radiol Endod. 2001;92:150-155.

9. Juodzbalys G, Daugela P. Mandibular third molar impaction: Review of literature and a proposal of a classification. J Oral Maxillofac Res. 2013;4:e1.

10. Quek SL, Tay CK, Tay KH, Toh SL, Lim KC. Pattern of third molar impaction in a Singapore Chinese population: a retrospective radiographic survey. Int J Oral Maxillofac Surg. 2003;32:548-552.

11. Yuasa H, Sugiura M. Clinical postoperative findings after removal of impacted mandibular third molars: prediction of postoperative facial swelling and pain based on preoperative variables. Br J Oral Maxillofac Surg. 2004;42:209-214.

12. Kim J, Ellis GL, Eisenberg E. Dental follicular tissue: misinterpretation as odontogenic tumors. J Oral Maxillofac Surg. 1993;51:762767

13. Edamatsu M, Kumamoto H, Ooya K, Echigo S. Apoptosis-related factors in the epithelial components of dental follicles and dentigerous cysts associated with impacted third molars of the mandible. Oral Surg Oral Med Oral Pathol Oral Radiol Endod. 2005;99:17-23.

14. Chu FC, Li TK, Lui VK, Newsome PR, Chow RL, Cheung LK. Prevalence of impacted teeth and associated pathologies: A radiographic study of Chinese population. Hong Kong Med J. 2003;9:158-163. 15. Haghanifar S, Moudi E, Seyedmajidi M, Mehdizadeh M, Nosrati $\mathrm{K}$, Abbaszadeh N, et al. Can the follicle-crown ratio of the impacted third molars be a reliable indicator of pathologic problem? J Dent (Shiraz). 2014;15:187-191.

16. Dula K, Bornstein M M, Buser D, Dagassan- Berndt D, Ettlin D A, Filippi A, et al. SADMFR guidelines for the use of cone-beam computed tomography/digital volume tomography. Swiss Dent J. 2014; 124:1169-1183.

17. Matzen LH, Hintze H, Spin-Neto R, Wenzel A. Reproducibility of mandibular third molar assessment comparing two cone beam CT units in a matched pairs design. Dentomaxillofac Radiol. 2013;42:20130228.

18. European Commission. Radiation protection 172. Evidence- based guidelines on cone beam CT for dental and maxillofacial radiology. Luxembourg: Office for Offical Publications of the European Communities; 2012. Available at: http://ec.europa.eu/energy/ nuclear/radiation_protection/publications_en.htm [accessed on May 21, 2012]

19. National Institute of Health. Removal of third molars. Sponsored by the National Institute of Dental Research. Natl Inst Health Consens Dev Conf Summ. 1979;2:65-68.

20. Quek S, Tay C, Tay K, Toh S, Lim K. Pattern of third molar impaction in a Singapore Chinese population: a retrospective radiographic survey. Int J Oral Maxillafac Surg. 2003;32:548-552.

21. Kaushal N, Soni S. Ameloblastoma in dental follicle of an asymptomatic impacted tooth. Indian J Oral Health. 2011;1:50-51.

22. Leitner C, Hoffman J, Kröber S, Reinert S. Low-grade malignant fibrosarcoma of the dental follicle of an unerupted third molar without clinical evidence of any follicular lesion. J Oral Maxillofac Surg. 2007;35:48-51.

23. Adelsperger J, Campbell JH, Coates DB, Summerlin DJ, Tomich CE. Early soft tissue pathosis associated with impacted third molars without pericoronal radiolucency. Oral Surg Oral Med Oral Pathol Oral Radiol Endod. 2000;89:402-406.

24. White SC, Pharaoh MJ. Oral radiology principals and interpretations 5th ed. St Louis: Mosby; 2004.

25. Duarte BG, Assis D, Ribeiro-Júnior P, Gonçales ES. Does the relationship between retained mandibular third molar and mandibular angle fracture exist? An assessment of three possible causes. Craniomaxillofac Trauma Reconstruction. 2012;5:127-135.

26. Chen L, Shaw C, Altunbas M, Lai C, Liu X. Spatial resolution properties in cone beam CT: a simulation study. Med Phys. 2008;35:724734.

27. Santos T, Cordeiro J, Raimundo R, Frazão M, Gomes A. Relação topográfica entre o canal mandibular e o terceiro molar inferior em tomografias de feixe volumétrico. Rev Cir Traumatol Buco-Maxilo-Fac. 2009;9:79-88.

28. Costa A, Sousa S, Grappo S, Haiter F. Mesial inclination of impacted third molars and its propensity to stimulate external root resorption in second molars: A cone-beam computed tomographic evaluation. J Oral Maxillofac Surg. 2015;73:379-386.

29. Nunn M, Fish M, Garcia R, Kaye E, Figueroa R, Gohel A, Miyamoto T. Response to Letter to the Editor, "Retained Asymptomatic Third Molars and Risk for Second Molar Pathology." Journal of Dental Research. 2014;93:320-321.

Acknowledgments

We would like to thank Dr. Hugo Aguayo and CDI Diagnostic Imaging for their guidance and interest in our study.

Conflict of Interest

The authors declare that they have no conflict of interest. 